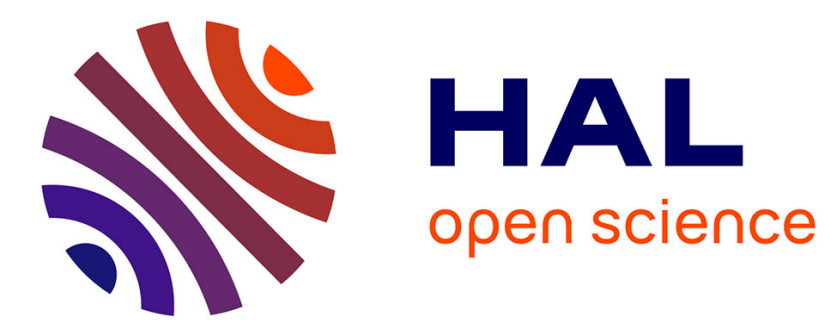

\title{
Reilly-type spinorial inequalities
}

Nicolas Ginoux

\section{To cite this version:}

Nicolas Ginoux. Reilly-type spinorial inequalities. Mathematische Zeitschrift, 2002, 241 (3), pp.513525. 10.1007/s00209-002-0428-4 . hal-03117213

\section{HAL Id: hal-03117213 https://hal.science/hal-03117213}

Submitted on 21 Jan 2021

HAL is a multi-disciplinary open access archive for the deposit and dissemination of scientific research documents, whether they are published or not. The documents may come from teaching and research institutions in France or abroad, or from public or private research centers.
L'archive ouverte pluridisciplinaire $\mathbf{H A L}$, est destinée au dépôt et à la diffusion de documents scientifiques de niveau recherche, publiés ou non, émanant des établissements d'enseignement et de recherche français ou étrangers, des laboratoires publics ou privés. 


\title{
Reilly-type spinorial inequalities
}

\author{
Nicolas Ginoux
}

January 21, 2021

Institut Élie Cartan, Université Henri Poincaré, Nancy I, B.P. 239, 54506 Vandœuvre-Lès-Nancy Cedex, France

E-mail: ginoux@iecn.u-nancy.fr

Key-words: Dirac operator, conformal geometry, spectrum, hypersurfaces

AMS subject classification: Differential geometry, global analysis, 53C27, 53C40, 53C 80, 58G25

ABSTRACT: We give a new extrinsic upper bound for the smallest eigenvalues of the Dirac operator of a hypersurface. If the ambient manifold is the hyperbolic space, we show that its limiting case is achieved only for geodesic spheres.

Let $(M, g)$ be an $m$-dimensional compact Riemannian manifold isometrically immersed in one of the three space-forms $\widetilde{M}=\mathbb{R}^{m+1}, S^{m+1}$ or $\mathbb{H}^{m+1}$. Consider the problem of finding sharp upper bounds for the smallest eigenvalue $\lambda_{1}$ of the Dirac operator of $M$.

Such a problem has been examined by different authors (see [2], [5], [8], [3]). Recently, C. Bär gave upper bounds involving the $L^{2}$-norm of the mean curvature $H$ of the immersion as well as the ambient scalar curvature. More precisely, he showed that ([3], Theorem 4.1):

$$
\begin{array}{ll}
\lambda_{1}^{2} \leq \frac{m^{2}}{4 \operatorname{Vol}(M)} \int_{M} H^{2} v_{g}, & \text { if } \widetilde{M}=\mathbb{R}^{m+1}, \quad \text { and } \\
\lambda_{1}^{2} \leq \frac{m^{2}}{4 \operatorname{Vol}(M)} \int_{M}\left(H^{2}+1\right) v_{g}, & \text { if } \widetilde{M}=S^{m+1},
\end{array}
$$

with equality achieved for geodesic spheres. However, if $M$ is immersed in $\mathbb{H}^{m+1}$, C. Bär proved that ([3], Theorem 4.4):

$$
\left|\lambda_{1}\right| \leq \frac{m}{2}\left(1+\|H\|_{\infty}\right)
$$

which is not sharp: for geodesic spheres of radius $r$, the inequality is strict, and we have ([3], p. 590) $\lim _{r \rightarrow 0} \frac{2\left|\lambda_{1}\right|}{m\left(1+\|H\|_{\infty}\right)}=1$. Another estimate in terms of the $L^{2}$-norm of the mean curvature and the extrinsic radius of the hypersurface was given by the same author ([3], 
p.587), but the limiting-case could not be achieved.

In this paper, we investigate this case and prove the following:

Theorem 1 Assume that $M$ is isometrically immersed in $\mathbb{H}^{m+1}$, seen as the upper halfsphere carrying the metric $g=e^{2 u} g_{0}$ conformal to the standard metric $g_{0}$ on $S^{m+1}$. Then

$$
\lambda_{1}^{2} \leq \frac{m^{2}}{4 \operatorname{Vol}(M)} \int_{M}\left(H^{2}-1\right) v_{g}+\frac{1}{4 \operatorname{Vol}(M)}\|d u\|_{L^{2}(M)}^{2},
$$

where $d u:=d\left(u_{\left.\right|_{M}}\right)$. Besides, if equality is achieved, then the function $u$ is constant on $M$, and therefore $M$ is a geodesic sphere.

In section 1, we recall basic facts regarding restricted spinor bundles. Then, applying the Min-Max principle, we first prove in section 2 a general estimate when the ambient manifold admits a real Killing spinor for a conformal change of the metric. Choosing this spinor as test-spinor in the Rayleigh quotient constitutes the key-point of the paper: real Killing spinors play the same role for the Dirac operator as coordinate functions do for the scalar Laplacian, they are the most suitable test-sections for our problem. The three problems can be uniformly treated, since the three space-forms can be conformally embedded in $S^{m+1}$, which admits real Killing spinors. The approach El Soufi and Ilias used for the scalar Laplacian in [9] can therefore be extended to the fundamental Dirac operator.

I thank Helga Baum, Oussama Hijazi and Sebastián Montiel for their support during the preparation of this paper. I also thank Bertrand Morel and Emmanuel Humbert for fruitful discussions and their careful reading of the paper.

\section{Spinors and Dirac operators on an oriented hyper- surface}

For preliminaries on spinors and Dirac operators, we refer to [13], [7], [10], [6], and [15]. For a recent review of the topic, see [14]. Let $\iota: M \longrightarrow(\widetilde{M}, g)$ be an immersion from a connected and oriented hypersurface $M$ into a Riemannian spin manifold $(\widetilde{M}, g)$. We shall always assume that the dimension $m$ of $M$ is greater than or equal to 2 . Let $\nu: M \longrightarrow$ $N M$ be the unitary section of the normal bundle $N M$ such that, for every positivelyoriented basis $\left(X_{1}, \ldots, X_{m}\right)$ of $T M$, the basis $\left(X_{1}, \ldots, X_{m}, \nu\right)$ of $\left.T \widetilde{M}\right|_{M}$ is positivelyoriented. Let $B$ be the shape-operator of the immersion $\iota$, seen as a field of symmetric endomorphisms of $T M$. We equip $M$ with the induced metric and denote it by $g$, with the associated norm $|\cdot|$, the volume element $v_{g}$ and the total volume $\operatorname{Vol}(M)$. We respectively denote by $\nabla$ and $\widetilde{\nabla}$ the Levi-Civita connections of $(M, g)$ and $(\widetilde{M}, g)$.

The normal bundle being trivialized by $\nu$, the manifold $(M, g)$ admits an induced spinstructure. We denote by $\Sigma M$ (resp. $\Sigma \widetilde{M}$ ) the vector bundle of spinors of $(M, g)$ (resp. 
of $(\widetilde{M}, g))$. We set

$$
\Sigma= \begin{cases}\Sigma M & \text { if } m \text { is even } \\ \Sigma^{0} M \oplus \Sigma^{1} M & \text { if } m \text { is odd }\end{cases}
$$

where, for $m$ odd and $j \in\{0,1\}, \Sigma^{j} M$ is the vector bundle of spinors on $M$ on which the complex volume form acts by $(-1)^{j} \mathrm{Id}$. We denote by ' ${ }_{M}$ ' (resp. ' ') the Clifford multiplication on $\Sigma\left(\right.$ resp. on $\left.\left.\Sigma \widetilde{M}\right|_{M}\right)$.

The vector bundle $\Sigma$ can be endowed with a Hermitian inner product, denoted by $\langle\cdot, \cdot\rangle$, and a covariant derivative $\nabla$, which satisfy the following properties, for all vector fields $X$ and $Y$ on $M$ and sections $\psi$ and $\phi$ of $\Sigma$ :

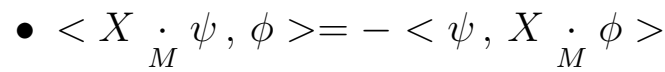

- $X(<\psi, \phi>)=<\nabla_{X} \psi, \phi>+<\psi, \nabla_{X} \phi>$

- $\nabla_{X}\left(Y_{\dot{M}}^{\dot{*}} \psi\right)=\nabla_{X} Y_{\dot{M}}^{\dot{m}} \psi+Y_{\dot{M}} \nabla_{X} \psi$.

The vector bundle $\Sigma \widetilde{M}$ (and therefore $\left.\Sigma \widetilde{M}\right|_{M}$ ) can also be endowed with a covariant derivative $\widetilde{\nabla}$ and a Hermitian inner product satisfying the same properties.

Now there exists an isomorphism between $\Sigma$ and $\left.\Sigma \widetilde{M}\right|_{M}$,

$$
\begin{aligned}
& \left.\Sigma \longrightarrow \Sigma \widetilde{M}\right|_{M} \\
& \psi \longmapsto \Psi,
\end{aligned}
$$

which satisfies, for every vector field $X$ on $M$ and for every section $\psi$ of $\Sigma$ :

- $X_{M} \underset{\dot{M}}{ } \psi$ is mapped onto $X \cdot \nu \cdot \Psi$.

- $\psi \longmapsto \Psi$ is a unitary isomorphism w.r.t. the respective Hermitian inner products

- w.r.t. the respective covariant derivatives $\nabla$ and $\widetilde{\nabla}$ :

$$
\widetilde{\nabla}_{X} \Psi=\nabla_{X} \psi+\frac{1}{2} B(X) \cdot \nu \cdot \Psi
$$

The Hermitian inner products on $\Sigma$ and $\left.\Sigma \widetilde{M}\right|_{M}$ will hence be denoted by the same symbol, with associated norm ' $|\cdot|$ '. Note however that this isomorphism is not unique, and that it doesn't preserve the covariant derivatives as can be seen in (2).

The isomorphism (1) can also be chosen so that the action of $i \nu$ on $\left.\Sigma \widetilde{M}\right|_{M}$ induces an endomorphism on $\Sigma$, denoted by $A$, defined for every $\psi$ in $\Sigma$ by:

$$
i \nu \cdot \Psi=A(\psi) .
$$


The endomorphism $A$ satisfies, for every vector field $X$ on $M$ and every section $\psi$ of $\Sigma$ :

$$
\left\{\begin{array}{l}
A^{2}=I d \\
|A(\psi)|=|\psi| \\
A(X \cdot \psi)=-X \cdot A(\psi) \\
\nabla_{X}(A(\psi))=A\left(\nabla_{X} \psi\right)
\end{array}\right.
$$

If $m$ is even, the vector bundle $\Sigma$ splits under the action of the complex volume form into $\Sigma=\Sigma^{+} \oplus \Sigma^{-}$, and $A$ is given by:

$$
A\left(\psi^{+}+\psi^{-}\right)=\psi^{+}-\psi^{-}, \text {where } \psi^{ \pm} \in \Sigma^{ \pm} .
$$

If $m$ is odd, and if $\tau: \Sigma^{0} M \longrightarrow \Sigma^{1} M$ is the isomorphism given by the equivalence of the spin representations, then

$$
A=\left(\begin{array}{ll}
0 & \tau^{-1} \\
\tau & 0
\end{array}\right)
$$

In a local orthonormal basis $\left(X_{j}\right)_{1 \leq j \leq m}$ of $T M$, let $D$ and $\widetilde{D}$ be the operators defined by:

$$
D=\sum_{j=1}^{m} X_{j}{ }_{M} \nabla_{X_{j}}, \quad \widetilde{D}=\sum_{j=1}^{m} X_{j} \cdot \widetilde{\nabla}_{X_{j}} .
$$

The operator $\widetilde{D}$ is called the Dirac-Witten operator (Cf [16]). These two operators, acting respectively on sections of $\Sigma$ and $\left.\Sigma \widetilde{M}\right|_{M}$, are related by

$$
\forall \psi \in \Gamma(\Sigma), \quad \widetilde{D} \Psi=\nu \cdot\left(D \psi-\frac{m H}{2} \Psi\right)
$$

where $H=\frac{1}{m} \sum_{j=1}^{m} g\left(B\left(X_{j}\right), X_{j}\right)$ is the mean curvature of the immersion $\iota$.

Note 1 If $m$ is even, the operator $D$ coincides with the Dirac operator $D_{M}$ of the manifold $(M, g)$. However, if $m$ is odd, via the isomorphism $\Sigma^{0} M \stackrel{\tau}{\longrightarrow} \Sigma^{1} M$, calling $D_{M}$ the Dirac operator of $(M, g)$ acting on the sections of $\Sigma^{0} M$,

$$
D=D_{M} \oplus-D_{M},
$$

that is to say

$$
\forall \phi^{0}, \psi^{0} \in \Gamma\left(\Sigma^{0} M\right), \quad D\left(\phi^{0} \oplus \tau\left(\psi^{0}\right)\right)=D_{M} \phi^{0} \oplus-\tau\left(D_{M} \psi^{0}\right) .
$$

When $M$ is compact, if $(\cdot, \cdot):=\int_{M}<\cdot, \cdot>v_{g}$, with associated norm $\|\cdot\|$, the operator $D$ is formally self-adjoint with respect to $(\cdot, \cdot)$ :

$$
\forall \psi, \phi \in \Gamma(\Sigma), \quad(D \psi, \phi)=(\psi, D \phi) .
$$

The eigenvalues of $D$ are therefore real. 
Lemma 1 The spectrum of $D$ is symmetric with respect to zero.

Proof: Just note that, for every section $\phi$ of $\Sigma$,

$$
D(A(\phi))=-A(D \phi),
$$

so if $\phi$ is an eigenspinor of $D$ for the eigenvalue $\lambda$, then $A(\phi)$ is an eigenspinor of $D$ for the eigenvalue $-\lambda$.

Note 2 If $m$ is odd, every eigenvalue of $D_{M}$ is an eigenvalue of $D$. Nevertheless, if $\lambda$ is an eigenvalue of $D$, then $\lambda$ or $-\lambda$ is an eigenvalue of $D_{M}$.

When $M$ is compact, the operator $D$ being elliptic, its eigenvalues form an increasing unbounded sequence. The sequence $\left(\lambda_{k}\right)_{k \geq 1}$ of eigenvalues of $D_{M}$ (counted with their multiplicities) will thereby be ordered with increasing absolute value:

$$
0 \leq\left|\lambda_{1}\right| \leq\left|\lambda_{2}\right| \leq \ldots \leq\left|\lambda_{k}\right| \leq\left|\lambda_{k+1}\right| \leq \ldots
$$

\section{Upper eigenvalue bounds when the ambient man- ifold admits a twistor-spinor}

We assume $M$ to be compact. In order to obtain upper bounds for the smallest eigenvalues of $D$, we shall use the following well-known proposition:

Proposition 1 (Min-Max principle) For each positive integer $k$,

$$
\lambda_{k}^{2}=\underset{E_{k} \subset \Gamma(\Sigma)}{\operatorname{Min}}\left\{\operatorname{Max}_{\psi \in E_{k} \backslash\{0\}}\left\{\frac{\left(D^{2} \psi, \psi\right)}{\|\psi\|^{2}}\right\}\right\}
$$

where the minimum is taken over all the $k$-dimensional vector subspaces $E_{k}$ of $\Gamma(\Sigma)$.

We need, to apply the Min-Max principle, to pick a subspace $E_{k}$ of sections (called testsections) of $\Sigma$ on which will be computed the Rayleigh-quotient $\frac{\left(D^{2} \psi, \psi\right)}{\|\psi\|^{2}}$. The three modelspaces carry particular spinor fields whose restrictions to $M$ form natural candidates to be test-spinors, namely Killing spinors. Recall that, given a complex constant $\alpha$, an $\alpha$ Killing spinor on $(\widetilde{M}, g)$ is a non-zero section $\Psi$ of $\Sigma \widetilde{M}$ satisfying, for every vector field $Z$ on $\widetilde{M}$

$$
\widetilde{\nabla}_{Z} \Psi=\alpha Z \cdot \Psi
$$

If such a section exists, it can be shown that $\alpha$ must be real or purely imaginary, and the manifold $(\widetilde{M}, g)$ must be Einstein with constant scalar curvature equal to $4 m(m+1) \alpha^{2}$ 
([6], [10]). A Killing spinor has no zero since it is a parallel section for the covariant derivative $Z \mapsto \widetilde{\nabla}_{Z}-\alpha Z$. There are however big differences between real and imaginary Killing spinors, particularly concerning the length-function $|\Psi|$ of such a spinor: this function is constant on $\widetilde{M}$ when $\alpha$ is real, whereas it cannot be constant when $\alpha$ is imaginary. This is the first reason why we would be more interested in computing the Rayleigh-quotient on real Killing spinors rather than on imaginary ones.

Both the Euclidean space and the round sphere admit real Killing spinors; these are parallel spinors on $\mathbb{R}^{m+1}$ (i.e. $\alpha=0$ ), and $\pm \frac{1}{2}$-Killing spinors on $S^{m+1}$. By computing the Rayleigh-quotient with such test-spinors C. Bär obtained his sharp upper bounds for hypersurfaces of $\mathbb{R}^{m+1}$ or $S^{m+1}$ ([3], corollaries 4.2 and 4.3 ).

Conversely, the hyperbolic space admits $\pm \frac{i}{2}$-Killing spinors. How can one then choose a real Killing spinor as test-spinor? The idea is as follow: embed $\mathbb{H}^{m+1}$ in $S^{m+1}$, compare the spinors for two conformal metrics, and use the restriction of a real Killing spinor on the sphere to the hypersurface $M \hookrightarrow \mathbb{H}^{m+1}$ as test-spinor.

We first recall basic facts about spinors and conformal metrics (see [13], [7], [4], [12] or [11]). Let $\bar{g}=e^{2 u} g$ be a conformal change of the metric on $\widetilde{M}$, where $u$ is a smooth function on $M$. The manifold $(\widetilde{M}, \bar{g})$ being spin, let $\Sigma_{\bar{g}} \widetilde{M}$ be its vector bundle of spinors, and denote by "-." Clifford multiplication on $\Sigma_{\bar{g}} \widetilde{M}$. There exists an isometry,

$$
\begin{aligned}
\Sigma \widetilde{M} & \longrightarrow \Sigma_{\bar{g}} \widetilde{M} \\
\Phi & \longmapsto \bar{\Phi}
\end{aligned}
$$

satisfying, for every vector field $Z$ on $\widetilde{M}$ and every section $\Phi$ in $\Sigma \widetilde{M}$,

$$
\overline{Z \cdot \Phi}=e^{-u} Z \cdot \bar{\Phi} .
$$

Such an isometry exists as well from $\Sigma$ onto $\Sigma_{\bar{g}}$, which will be denoted similarly.

With respect to the covariant derivatives $\nabla$ and $\nabla^{\bar{g}}$ on $\Sigma$ and $\Sigma_{\bar{g}}$ respectively, the following relation holds:

$$
\nabla_{X}^{\bar{g}} \bar{\phi}=\overline{\nabla_{X} \phi}-\frac{1}{2} \overline{X_{M} \cdot d u_{M} \phi}-\frac{1}{2} d u(X) \bar{\phi},
$$

for every vector field $X$ on $M$ and every section $\phi$ of $\Sigma$. An analogous relation holds for the covariant derivatives $\widetilde{\nabla}$ and $\widetilde{\nabla}^{\bar{g}}$ : for every vector field $X$ on $M$, and every section $\Phi$ of $\left.\Sigma \widetilde{M}\right|_{M}$,

$$
\widetilde{\nabla}_{X}^{\bar{g}} \bar{\Phi}=\overline{\widetilde{\nabla}_{X} \Phi}-\frac{1}{2} \overline{X \cdot d u \cdot \Phi}-\frac{\nu(u)}{2} \overline{X \cdot \nu \cdot \Phi}-\frac{1}{2} d u(X) \bar{\Phi}
$$

(remember that $d u=d\left(u_{\left.\right|_{M}}\right)$ ).

\subsection{Main result}

When the ambient manifold $\widetilde{M}$ carries an $N$-dimensional vector space of $\alpha$-Killing spinors for a certain metric, define

$$
\mu(N):= \begin{cases}N & \text { if } m \text { is even } \\ {\left[\frac{N+1}{2}\right]} & \text { if } m \text { is odd }\end{cases}
$$


(where $[\cdot]$ is the integer part).

Theorem 2 Assume that, for a conformal change of the metric $\bar{g}=e^{2 u} g$, the manifold $(\widetilde{M}, \bar{g})$ admits an $N$-dimensional vector space of real Killing spinors. Then, for $1 \leq k \leq$ $\mu(N)$,

$$
\lambda_{k}^{2} \leq \frac{m^{2}}{4 \operatorname{Vol}(M)} \int_{M}\left(H^{2}+R(\iota)\right) v_{g}+\frac{1}{4 \operatorname{Vol}(M)}\|d u\|_{L^{2}(M)}^{2},
$$

where, in a local orthonormal basis $\left(X_{j}\right)_{1 \leq j \leq m}$ of $T M$,

$$
R(\iota):=\frac{1}{m(m-1)} \sum_{1 \leq i \neq j \leq m} \tilde{K}\left(X_{i}, X_{j}\right),
$$

and $\widetilde{K}$ denotes the sectional curvature of $(\widetilde{M}, g)$.

Proof: Let $\bar{\Psi}$ be an $\alpha$-Killing spinor on $(\widetilde{M}, \bar{g})$ ( $\alpha$ is assumed to be real). Compute the Rayleigh-quotient $\frac{\left(D^{2} \psi, \psi\right)}{\|\psi\|^{2}}$, where, as in Section $1, \psi$ is the preimage of $\Psi$ through the isomorphism (1). For this purpose, we need first to compare the squares of $D$ and $\widetilde{D}$, then relate the Dirac-Witten operators $\widetilde{D}$ and $\widetilde{D}_{\bar{g}}$ for metrics $g$ and $\bar{g}$ respectively.

Lemma 2 For every section $\phi$ of $\Sigma$, we have:

1. $\widetilde{D}^{2} \Phi=D^{2} \phi-\frac{m}{2} d H \cdot \nu \cdot \Phi-\frac{m^{2} H^{2}}{4} \Phi$

2. $\widetilde{D}_{\bar{g}} \bar{\Phi}=e^{-u}\left(\overline{\widetilde{D} \Phi}+\frac{m-1}{2} \overline{d u \cdot \Phi}+\frac{m}{2} \nu(u) \overline{\nu \cdot \Phi}\right)$.

Proof: For every section $\phi$ in $\Sigma$, and in a local orthonormal basis $\left(X_{j}\right)_{1 \leq j \leq m}$ of $T M$,

$$
\begin{aligned}
\widetilde{D}(\nu \cdot \Phi) & =\sum_{j=1}^{m} X_{j} \cdot \widetilde{\nabla}_{X_{j}} \nu \cdot \Phi+X_{j} \cdot \nu \cdot \widetilde{\nabla}_{X_{j}} \Phi \\
& =m H \Phi-\nu \cdot \widetilde{D} \Phi .
\end{aligned}
$$

From $\widetilde{D} \Phi=\nu \cdot\left(D \phi-\frac{m H}{2} \Phi\right)$ and this identity, we deduce that:

$$
\begin{aligned}
\widetilde{D}^{2} \Phi & =m H\left(D \phi-\frac{m H}{2} \Phi\right)-\nu \cdot \widetilde{D}\left(D \phi-\frac{m H}{2} \Phi\right) \\
& =m H\left(D \phi-\frac{m H}{2} \Phi\right)-\nu \cdot \nu \cdot\left(D^{2} \phi-\frac{m}{2} D(H \phi)-\frac{m H}{2} D \phi+\frac{m^{2} H^{2}}{4} \Phi\right) \\
& =m H\left(D \phi-\frac{m H}{2} \Phi\right)+D^{2} \phi-\frac{m}{2} d H \cdot \nu \cdot \Phi-\frac{m H}{2} D \phi-\frac{m H}{2} D \phi+\frac{m^{2} H^{2}}{4} \Phi \\
& =D^{2} \phi-\frac{m}{2} d H \cdot \nu \cdot \Phi-\frac{m^{2} H^{2}}{4} \Phi .
\end{aligned}
$$


For the second point of Lemma 2, we apply formula (3) and use the fact that, if $\left(X_{j}\right)_{1 \leq j \leq m}$ is a local $g$-orthonormal basis of $T M$, then $\left(e^{-u} X_{j}\right)_{1 \leq j \leq m}$ is a local $\bar{g}$-orthonormal basis of $T M$ :

$$
\begin{aligned}
\widetilde{D}_{\bar{g}} \bar{\Phi} & =\sum_{j=1}^{m} e^{-2 u} X_{j}: \widetilde{\nabla}_{X_{j}}^{\bar{g}} \bar{\Phi} \\
& =e^{-u}\left(\sum_{j=1}^{m} \overline{X_{j} \cdot \widetilde{\nabla}_{X_{j}} \Phi}-\frac{1}{2} \overline{X_{j} \cdot X_{j} \cdot d u \cdot \Phi}-\frac{\nu(u)}{2} \overline{X_{j} \cdot X_{j} \cdot \nu \cdot \Phi}+\frac{1}{2} \overline{d u\left(X_{j}\right) X_{j} \cdot \Phi}\right) \\
& =e^{-u}\left(\overline{\widetilde{D} \Phi}+\frac{m-1}{2} \overline{d u \cdot \Phi}+\frac{m \nu(u)}{2} \overline{\nu \cdot \Phi}\right)
\end{aligned}
$$

We deduce from the second part of Lemma 2 that

$$
\widetilde{D} \Psi=-m \alpha e^{u} \Psi-\frac{m-1}{2} d u \cdot \Psi-\frac{m}{2} \nu(u) \nu \cdot \Psi .
$$

Now compute $\widetilde{D}^{2} \Psi$ :

$$
\begin{aligned}
\widetilde{D}\left(e^{u} \Psi\right) & =d\left(e^{u}\right) \cdot \Psi+e^{u} \widetilde{D} \Psi \\
& =-m \alpha e^{2 u} \Psi-\frac{m-3}{2} e^{u} d u \cdot \Psi-\frac{m}{2} e^{u} \nu(u) \nu \cdot \Psi .
\end{aligned}
$$

On the other hand, calling $\triangle$ the scalar Laplacian on $(M, g)$,

$$
\begin{aligned}
\widetilde{D}(d u \cdot \Psi) & =\sum_{j=1}^{m} X_{j} \cdot \widetilde{\nabla}_{X_{j}} d u \cdot \Psi+\sum_{j=1}^{m} X_{j} \cdot d u \cdot \widetilde{\nabla}_{X_{j}} \Psi \\
& =\sum_{j=1}^{m} X_{j} \cdot \nabla_{X_{j}} d u \cdot \Psi+\sum_{j=1}^{m} g\left(B(d u), X_{j}\right) X_{j} \cdot \nu \cdot \Psi-d u \cdot \sum_{j=1}^{m} X_{j} \cdot \widetilde{\nabla}_{X_{j}} \Psi \\
& -2 \sum_{j=1}^{m} d u\left(X_{j}\right) \widetilde{\nabla}_{X_{j}} \Psi \\
& =(\triangle u) \Psi+B(d u) \cdot \nu \cdot \Psi-d u \cdot \widetilde{D} \Psi-2 \widetilde{\nabla}_{d u} \Psi .
\end{aligned}
$$

But, from (3),

$$
\widetilde{\nabla}_{d u} \Psi=\alpha e^{u} d u \cdot \Psi+\frac{1}{2} \nu(u) d u \cdot \nu \cdot \Psi
$$

hence

$\widetilde{D}(d u \cdot \Psi)=(\triangle u) \Psi+B(d u) \cdot \nu \cdot \Psi+(m-2) \alpha e^{u} d u \cdot \Psi-\frac{m-1}{2}|d u|^{2} \Psi+\frac{m-2}{2} \nu(u) d u \cdot \nu \cdot \Psi$.

Besides,

$$
\begin{aligned}
\widetilde{D}(\nu(u) \nu \cdot \Psi) & =d(\nu(u)) \cdot \nu \cdot \Psi+m H \nu(u) \Psi-\nu(u) \nu \cdot \widetilde{D} \Psi \\
& =d(\nu(u)) \cdot \nu \cdot \Psi+m H \nu(u) \Psi+m \alpha e^{u} \nu(u) \nu \cdot \Psi \\
& -\frac{m-1}{2} \nu(u) d u \cdot \nu \cdot \Psi-\frac{m}{2} \nu(u)^{2} \Psi .
\end{aligned}
$$


We then have:

$$
\begin{aligned}
\widetilde{D}^{2} \Psi & =m^{2} \alpha^{2} e^{2 u} \Psi-\alpha e^{u} d u \cdot \Psi-\frac{m-1}{2} B(d u) \cdot \nu \cdot \Psi+\frac{(m-1)^{2}}{4}|d u|^{2} \Psi \\
& -\frac{m-1}{2}(\triangle u) \Psi+\frac{m-1}{2} \nu(u) d u \cdot \nu \cdot \Psi-\frac{m}{2} d(\nu(u)) \cdot \nu \cdot \Psi \\
& -\frac{m^{2} H}{2} \nu(u) \Psi+\frac{m^{2} \nu(u)^{2}}{4} \Psi .
\end{aligned}
$$

The first part of Lemma 2 yields:

$$
\begin{aligned}
D^{2} \psi & =\frac{m^{2}}{4}\left(H^{2}-2 H \nu(u)+\nu(u)^{2}+4 \alpha^{2} e^{2 u}\right) \Psi+\frac{(m-1)^{2}}{4}|d u|^{2} \Psi \\
& -\frac{m-1}{2}(\triangle u) \Psi+X_{0} \cdot \Psi+Y_{0} \cdot \nu \cdot \Psi,
\end{aligned}
$$

where $X_{0}$ and $Y_{0}$ are the tangent vector fields on $M$ defined by:

$$
\begin{aligned}
X_{0} & :=-\alpha e^{u} d u \\
Y_{0} & :=\frac{m-1}{2}(\nu(u) d u-B(d u))+\frac{m}{2} d(H-\nu(u)) .
\end{aligned}
$$

Note that, if $H_{\bar{g}}$ is the mean curvature of the immersion $\iota: M \longrightarrow(\widetilde{M}, \bar{g})$, then

$$
\begin{aligned}
H^{2}-2 H \nu(u)+\nu(u)^{2} & =(H-\nu(u))^{2} \\
& =e^{2 u} H_{\bar{g}}^{2} .
\end{aligned}
$$

According to A. El Soufi et S. Ilias ([9], Proposition 2),

$$
e^{2 u}\left(H_{\bar{g}}^{2}+\bar{R}(\iota)\right)=H^{2}+R(\iota)-\frac{m-2}{m}|d u|^{2}+\frac{2}{m} \triangle u,
$$

with $\bar{R}(\iota)=R(\iota, \bar{g})$.

But, if $(\widetilde{M}, g)$ is Einstein with scalar curvature equal to $\rho$, then $R(\iota)=\frac{\rho}{m(m+1)}$. Hence, in our case, $\bar{R}(\iota)=4 \alpha^{2}$, and relation (5) yields:

$$
D^{2} \psi=\frac{m^{2}}{4}\left(H^{2}+R(\iota)\right) \Psi+\frac{1}{4}|d u|^{2} \Psi+\frac{1}{2}(\triangle u) \Psi+X_{0} \cdot \Psi+Y_{0} \cdot \nu \cdot \Psi .
$$

Since $\bar{\Psi}$ has constant length on $\widetilde{M}$, the spinor $\psi$ is also of constant length on $M$, which we assume to be equal to 1 . Taking the Hermitian product of equality (6) with $\Psi$ gives:

$$
<D^{2} \psi, \psi>=\frac{m^{2}}{4}\left(H^{2}+R(\iota)\right)+\frac{1}{4}|d u|^{2}+\frac{1}{2} \triangle u+<X_{0} \cdot \Psi, \Psi>+<Y_{0} \cdot \nu \cdot \Psi, \Psi>.
$$

Since $X_{0}$ and $Y_{0}$ are tangent vector fields on $M$, the last two terms are purely imaginary. As the operator $D$ is formally self-adjoint, we therefore don't need these terms to compute the Rayleigh-quotient. Integrating over $M$, we obtain:

$$
\frac{\left(D^{2} \psi, \psi\right)}{(\psi, \psi)}=\frac{m^{2}}{4 \operatorname{Vol}(M)} \int_{M}\left(H^{2}+R(\iota)\right) v_{g}+\frac{1}{4 \operatorname{Vol}(M)} \int_{M}|d u|^{2} v_{g} .
$$

This holds for every $\alpha$-Killing spinor $\bar{\Psi}$ on $(\widetilde{M}, \bar{g})$. The Min-Max principle thus yields the result. 


\section{Notes}

1. If the function $u$ is constant on $\widetilde{M}$, i.e. if the manifold $(\widetilde{M}, g)$ itself admits $\alpha$-Killing spinors with real $\alpha$, the inequality (4) is just the one C. Bär proved in [3] (Theorem 4.1), since in this case $R(\iota)=4 \alpha^{2}$.

2. In case $m=2$, the integral $\int_{M}\left(H^{2}+R(\iota)\right) v_{g}$ is the so-called Willmore integral. This integral is invariant under conformal changes of the metric on $\widetilde{M}$.

3. When the ambient manifold $\widetilde{M}$ is simply connected, the spin structure of $(\widetilde{M}, g)$ is preserved under the action of orientation-preserving conformal diffeomorphisms. We can therefore improve the inequality (4): for $k \in\{1, \ldots, \mu(N)\}$,

$$
\lambda_{k}^{2} \leq \frac{m^{2}}{4 \operatorname{Vol}(M)} \int_{M}\left(H^{2}+R(\iota)\right) v_{g}+\frac{1}{4 \operatorname{Vol}(M)} \inf \left\{\|d v\|^{2}\right\},
$$

where the infimum is taken over all conformal diffeomorphisms $\gamma$ of $(\widetilde{M}, g)$ and functions $v$ are given by: $e^{2 v} g=\gamma^{*} \bar{g}$. From the Gauß equation, this inequality is equivalent to

$$
\lambda_{k}^{2} \leq \frac{m}{4(m-1) \operatorname{Vol}(M)} \int_{M} S v_{g}+\frac{m}{4(m-1) \operatorname{Vol}(M)}\|\tau(\iota)\|^{2}+\frac{1}{4 \operatorname{Vol}(M)} \inf \left\{\|d v\|^{2}\right\} .
$$

Compare this result to a similar one obtained by I. Agricola and T. Friedrich when $m=2$ (see [1], Theorem 2).

Now we examine the limiting-case in (4) for the eigenvalue $\lambda_{1}$.

Theorem 3 If $(\widetilde{M}, \bar{g})$ carries a maximal number of real non-parallel Killing spinors, then the limiting-case in (4) is achieved for the smallest eigenvalue $\lambda_{1}$ if and only if $d u=0$ and $H^{2}+R(\iota)$ is constant equal to $\frac{4 \lambda_{1}^{2}}{m^{2}}$.

Proof: If equality is achieved in (4) for $\lambda_{1}$, then for every real Killing spinor $\bar{\Psi}$ on $(\widetilde{M}, \bar{g})$, we have $D^{2} \psi=\lambda_{1}^{2} \psi$. But identity (6) yields

$$
\lambda_{1}^{2}=\frac{m^{2}}{4}\left(H^{2}+R(\iota)\right)+\frac{1}{4}|d u|^{2}+\frac{1}{2} \triangle u
$$

from which we deduce

$$
X_{0} \cdot \Psi+Y_{0} \cdot \nu \cdot \Psi=0 .
$$

If there exists a maximal number of $\alpha$-Killing spinors on $(\widetilde{M}, \bar{g})$ (i.e. $N=2^{\left[\frac{m+1}{2}\right]}$ ), then $X_{0}$ and $Y_{0}$ vanish identically on $M$. Indeed, fix a point $x$ in $M$, and let $\Phi$ be in $\left.\Sigma_{x} \widetilde{M}\right|_{M}$. There exists a Killing spinor $\bar{\Psi}$ on $(\widetilde{M}, \bar{g})$ such that $\Psi_{x}=\Phi$. The preceding identity 
says therefore that $X_{0}(x) \cdot \Phi+Y_{0}(x) \cdot \nu_{x} \cdot \Phi=0$ is valid for every $\Phi$ in $\left.\Sigma_{x} \widetilde{M}\right|_{M}$. The representation $\mathbb{C l}_{m+1} \longrightarrow \mathbb{C}\left(2^{\left[\frac{m+1}{2}\right]}\right)$ of the complex Clifford algebra $\mathbb{C l}_{m+1}$ being injective, we deduce that $X_{0}(x)+Y_{0}(x) \cdot \nu_{x}=0$, from which it follows that $X_{0}(x)=Y_{0}(x)=0$. Furthermore, if $\alpha \neq 0$, then $d u=0$ from the very definition of $X_{0}$.

Note If $\alpha \neq 0$, then in the limiting-case $\lambda_{1} \neq 0$ and $\psi$ cannot be an eigenspinor for $D$.

Example Let $\left(\mathbb{H}^{m+1}, g\right)$ be the $m+1$-dimensional hyperbolic space (of constant sectional curvature -1 ). Consider it as the upper half-sphere

$$
\left\{x=\left(x_{1}, \ldots, x_{m+2}\right) \in \mathbb{R}^{m+2}, \quad x_{1}^{2}+\ldots+x_{m+2}^{2}=1 \text { and } x_{m+2}>0\right\},
$$

carrying the metric $g=\frac{1}{x_{m+2}^{2}} g_{0}$, where $g_{0}$ is the standard metric on the round sphere $S^{m+1}$. Since $\left(S^{m+1}, g_{0}\right)$ carries a maximal number of real non-parallel Killing spinors, we can apply Theorem 2 and obtain: for $1 \leq k \leq 2^{\left[\frac{m}{2}\right]}$,

$$
\lambda_{k}^{2} \leq \frac{m^{2}}{4 \operatorname{Vol}(M)} \int_{M}\left(H^{2}-1\right) v_{g}+\frac{1}{4 \operatorname{Vol}(M)} \int_{M} g_{0}\left(e_{m+2}^{T}, e_{m+2}^{T}\right) v_{g},
$$

where $e_{m+2}^{T}$ is the orthogonal projection of vector $e_{m+2}=(0, \ldots, 0,1)$ in $\mathbb{R}^{m+2}$ onto the tangent space of $M$. Besides, if the limiting-case is achieved in this inequality, then, from Theorem 3 , the function $x_{m+2}$ is constant on $M$, i.e. $M$ is a geodesic sphere centered at $e_{m+2}$. Theorem 1 is hence proved.

Note that, because the group of isometries of $\left(\mathbb{H}^{m+1}, g\right)$ acts transitively on $\mathbb{H}^{m+1}$, every geodesic sphere satisfies:

$$
\lambda_{1}^{2}=\frac{m^{2}}{4}\left(H^{2}-1\right) .
$$

\section{References}

[1] I. Agricola and T. Friedrich, Upper bounds for the first eigenvalue of the Dirac operator on surfaces, J. Geom. Phys. 30 (1999), 1-22.

[2] N. Anghel, Extrinsic upper bounds for eigenvalues of Dirac-type operators, Proc. AMS (1993), 501-509.

[3] C. Bär, Extrinsic Bounds for Eigenvalues of the Dirac Operator, Ann. Glob. Anal. Geom. 16 (1998), 573-596.

[4] H. Baum, Spin-Strukturen und Dirac-Operatoren über pseudo-Riemannschen Mannigfaltigkeiten, Teubner-Texte zur Mathematik 41 (1981), Teubner-Verlag Leipzig.

[5] _ - An upper bound for the first eigenvalue of the Dirac operator on compact spin manifolds, Math. Zeit. 206 (1991), 409-422. 
[6] H. Baum, T. Friedrich, R. Grunewald, and I. Kath, Twistor and Killing Spinors on Riemannian Manifolds, Teubner Verlag, Stuttgart/Leipzig, 1991.

[7] J.P. Bourguignon O. Hijazi J.-L. Milhorat A. Moroianu, A Spinorial approach to Riemannian and Conformal Geometry, Monograph, (In preparation).

[8] U. Bunke, Upper bounds of small eigenvalues of the Dirac operator and isometric immersions, Ann. Glob. Anal. Geom. 9 (1991), 109-116.

[9] A. El Soufi S. Ilias, Une inégalité de type Reilly pour les sous-variétés de l'espace hyperbolique, Comment. Math. Helv. 672 (1992), 167-181.

[10] T. Friedrich, Dirac-Operatoren in der Riemannschen Geometrie, Adv. Lect. Math., 1997.

[11] O. Hijazi, Spectral properties of the Dirac operator and geometrical structures, Proceedings of the summer school on geometric methods in quantum field theory, July 12-30, 1999 (Villa de Leyva, Colombia), World Scientific, Physics, 2001.

[12] N. Hitchin, Harmonic spinors, Adv. in Math. 14 (1974), 1-55.

[13] H.B. Lawson M.L. Michelsohn, Spin Geometry, Princeton University Press, 1989.

[14] B. Morel, Eigenvalue Estimates for the Dirac-Schrödinger Operators, J. Geom. Phys. 38 (2001), 57-74.

[15] A. Trautman, The Dirac operator on hypersurfaces, Acta Phys. Polon. 6 (1995), $1283-1310$.

[16] X. Zhang, Lower bounds for eigenvalues of hypersurface Dirac operator, Math. Res. Letters 5 (1998), 199-210. 